\title{
dOENÇA CELIACA E O CONHECIMENTO dOS PROFISSIONAIS de saúde da ATENÇÃo PRIMÁRIA
}

\section{Celiac disease and knowledge of primary care health professionals}

\author{
Caroline Gonçalves Pustiglione Campos ${ }^{1}$, Aline Domingues Stumpfs Mendoza ${ }^{2}$, Elaine \\ Cristina Antunes Rinaldi ${ }^{3}$, Suellen Vienscoski Skupien ${ }^{4}$
}

\begin{abstract}
1. Enfermeira. Docente assistente do Curso Bacharelado em Enfermagem da Universidade Estadual de Ponta Grossa. ORCID: https://orcid.org/0000-0002-3803-6100

2. Enfermeira. Graduada em Enfermagem da Universidade Estadual de Ponta Grossa. ORCID: https://orcid. org/0000-0002-2173-6266

3. Enfermeira. Docente assistente do Curso Bacharelado em Enfermagem da Universidade Estadual de Ponta Grossa. ORCID: HTTP://orcid.org/0000-0002-2937-7405.

4. Enfermeira. Docente colaboradora do Curso Bacharelado em Enfermagem da Universidade Estadual de Ponta Grossa. ORCID:https://orcid.org/0000-0002-9503-6334
\end{abstract}

CONTATO: Caroline Gonçalves Pustiglione Campos | Teodoro Sampaio 421, oficinas PG. | CEP. 84035-640 | E-mail: carolgonc@hotmail.com

COMO CITAR: Campos CGP, Mendonza ADS, Rinaldi ECA, Skupien SV. Doença celíaca e o conhecimento dos profissionais de saúde da atenção primária. R. Saúde Públ. 2018 Dez;1(2):54-62

\footnotetext{
(c) (i) COPYRIGHT Esta obra é disponibilizada nos termos da Licença Creative Commons - 4. 0 Internacional. É permitida a reprodução parcial ou total desta obra, desde que citada a fonte.
}

RESUMO A doença celíaca é um problema de ordem mundial em decorrência da sua alta prevalência. O objetivo foi identificar o conhecimento dos enfermeiros, médicos e farmacêuticos da atenção primária sobre a doença celíaca. Trata-se de uma pesquisa quantitativa e descritiva. Realizada no município de Ponta Grossa no interior do Paraná em 19 unidades de saúde, com 82 profissionais, em 2016. A coleta de dados se deu pela aplicação de um questionário com perguntas fechadas, fundamentadas no Protocolo Clínico de Diretrizes Terapêuticas da Doença 
Celíaca. A análise dos dados foi por meio de tratamento estatístico. Resultou-se em 93,0\% dos profissionais de saúde responderam conhecer sobre a doença e 15,0\% relatam ter ciência do protocolo de atendimento ao paciente celíaco. Obteve-se 80,0\% de acertos referentes à sintomatologia típica e 40,0\% da forma atípica. Conclui-se há necessidade de capacitação por meio da educação em serviço, como estratégia de reforçar o manejo desta doença na atenção primária.

PALAVRAS-CHAVE: Doença Celíaca. Estearreia. Profissional da Saúde. Atenção Primária à Saúde.

ABSTRACT Celiac disease is a worldwide problem due to its high prevalence. The objective was to identify the knowledge of nurses, physicians and pharmacists from primary care to health about celiac disease. This is a quantitative and descriptive research. It was held in a municipality in the countryside of the state of Paraná in 19 health units, with 82 professionals, in 2016. Data collection was done through the application of a questionnaire with closed questions, based on the Clinical Protocol of Therapeutic Guidelines of Celiac Disease. Data analysis was conducted through statistical treatment. Ninety three percent of health professionals reported to have some knowledge about the disease, and $15.0 \%$ reported being aware of the protocol of care to the celiac patient. Eighty percent gave right answers for the typical symptomatology, and $40.0 \%$ for the atypical form. It was concluded that there is a need for training through in-service education, as a strategy to strengthen the management of this disease in primary care.

KEYWORDS: Celiac disease. Stearine. Health Professional. Primary Health Care.

\section{INTRODUÇÃO}

$\mathbf{A}$

doença celíaca é uma enteropatia crônica do intestino delgado, de caráter autoimune, causada pela exposição ao glúten em indivíduos propensos geneticamente. Trata-se de um processo inflamatório que envolve a mucosa do intestino delgado, levando à atrofia das vilosidades intestinais e, consequentemente, à má absorção, além de uma variedade de manifestações clínicas'

Como condição crônica, a doença celíaca possui as seguintes formas clínicas: clássica, não clássica, latente e assintomática. A forma clássica geralmente manifesta-se nos primeiros anos de vida apresentando sintomas como a diarreia ou constipação crônica, vômitos, emagrecimento, comprometimento variável do estado nutricional, dor e distensão abdominal. A forma não clássica é caracterizada pela ausência de sintomas digestivos, mas pode apresentar manifestações isoladas como baixa estatura, artrite, constipação intestinal, osteoporose e esterilidade. A latente é identificada através de biópsia jejunal normal, consumindo glúten, podendo também apresentar atrofia subtotal das vilosidades intestinais ${ }^{1-2}$.

A forma assintomática vem sendo reconhecida com maior frequência nas últimas duas décadas entre familiares de primeiro grau de pacientes celíacos por meio do desenvolvimento de marcadores sorológicos específicos. Aproximadamente 10,0\% dos parentes dos celíacos podem apresentar essa condição devido ao caráter familiar da desordem ${ }^{1-2}$.

A doença celíaca é um problema de ordem mundial em decorrência da sua alta prevalência, aproximadamente 1:100-300 pessoas em todo o mundo ${ }^{3}$. No Brasil, em um estudo realizado em 
Brasília foi encontrada uma prevalência de 0,3\% (1:294) para a doença celíaca, o que demonstra a significância de estudos no cenário brasileiro ${ }^{4}$.

Em conformidade com estudos, a prevalência da doença celíaca entre os países e em populações europeias ou de ancestralidade europeia varia de $0,3 \%$ a $1,0 \%$, e, provavelmente, muitos casos permanecem sem diagnóstico. No Brasil, os dados estatísticos oficiais são desconhecidos, portanto estima-se que existam 300 mil brasileiros portadores da doença, com maior incidência na região sudeste. Essa condição patológica é mais frequente em mulheres, numa proporção de 2:1 e atinge predominantemente os indivíduos de cor branca, entretanto, no Brasil, devido à miscigenação racial já foi descrita em mulatos ${ }^{5}$.

Aprovado pela Secretaria Nacional de Assistência à Saúde/Ministério da Saúde n 1149. de 11 de novembro de 2015, o Protocolo Clínico e Diretrizes Terapêuticas da Doença Celíaca menciona a falta de informação sobre a doença, e a dificuldade para o diagnóstico acabam prejudicando a adesão do paciente ao tratamento, limitando as possibilidades de melhora do quadro clínico.

A terapia nutricional recomendada é a retirada completa do glúten da dieta, o que faz com que os sintomas regridam e o estado nutricional seja restabelecido. A dieta imposta é restritiva, difícil e permanente, ocasionando alterações na rotina dos indivíduos e de sua família. 0 paciente após o diagnóstico passa a conviver com uma dieta restritiva devido à maioria dos produtos alimentícios conterem esta proteína na composição ${ }^{1-2}$.

Outro fator relevante para obediência à dieta é o conhecimento do paciente em relação à doença e seu tratamento, e, para isto, os profissionais de saúde têm a responsabilidade de esclarecê-los da forma mais detalhada possivel. Deve-se enfatizar que poucos estudos foram publicados avaliando a obediência à dieta e o conhecimento dos indivíduos que desenvolveram tal enfermidade ${ }^{6}$.

Os pacientes podem precisar, além dos cuidados médicos, do atendimento de outros profissionais de forma individualizada e coletiva. Enfatiza-se a necessidade da atenção multidisciplinar e multiprofissional a esses pacientes na atenção primária’'

As práticas de saúde e o processo de trabalho desenvolvido no âmbito das equipes têm colocado em debate a emergência das doenças crônicas como um importante problema de saúde pública, especialmente na atenção primária à saúde, ainda centrado em formas de organização que não se adéquam às necessidades de atenção a populações crescentemente assoladas por problemas de natureza crônica?.

Baseado nesses pressupostos, objetivouse identificar o conhecimento dos enfermeiros, médicos e farmacêuticos da atenção primária à saúde sobre o protocolo clínico da doença celíaca.

\section{METODOLOGIA}

Trata-se de pesquisa com abordagem quantitativa e descritiva realizada em unidades básicas de saúde no município de Ponta Grossa, Paraná.

O município de Ponta Grossa integra a Rede de Atenção Básica à Saúde, possuindo 52 Unidades de Saúde da Família, que abrigam 80 equipes de Estratégia Saúde da Família, 13 de Saúde Bucal e 02 Unidades Básicas, além de contar com aproximadamente 250 profissionais de saúde, dentre médicos e enfermeiros. A cobertura populacional do município pela Estratégia Saúde da Família é de 90\%, segundo competência de agosto de 2017 e tem como meta alcançar 95\% até $2020^{\circ}$.

Ressalta-se ainda que o município de Ponta Grossa possui aproximadamente 360.000 habitantes, sendo o perfil epidemiológico da população apresentado no Plano Municipal de Saúde, onde foram avaliados com base no diagnóstico situacional com finalidade de propor metas para reduzir: a Mortalidade MaternoInfantil por causas evitáveis; a Mortalidade por 
Causas Externas; a morbimortalidade por doenças crônicas não transmissíveis e por último o impacto decorrente dos transtornos mentais e do uso abusivo de álcool e outras drogas ${ }^{8}$.

Antes de iniciar a pesquisa foi realizado contato com o coordenador da Estratégia Saúde da Família, da Secretaria de Saúde do município, para identificar as Unidades Básicas que tinham no quadro de funcionários o profissional farmacêutico. Constatou-se apenas 09 Unidades Básicas e após foram acrescidas 10 unidades para compor a pesquisa, a fim de captar maior número de enfermeiros e médicos.

A amostra da pesquisa foi composta por 82 profissionais de saúde atuantes na atenção primária do município de Ponta Grossa, Paraná. Como critérios de inclusão na pesquisa têm-se: enfermeiros, médicos e farmacêuticos atuantes na atenção primária, com tempo de atuação mínimo de oito meses. Foram selecionadas 19 Unidades Básicas de Saúde, onde consta pelo menos uma de cada distrito sanitário do município.

O período de coleta de dados foi de julho a setembro de 2016, com aplicação de questionário estruturado, baseado no Protocolo Clínico e Diretrizes Terapêuticas da Doença Celíaca, contendo seis questões que contemplam os requisitos da assistência ao paciente celíaco.

A análise dos dados foi realizada por meio de tratamento estatístico, através do programa Excel ${ }^{\circledR}$ e expressos em frequência simples.

O estudo respeitou as exigências formais contidas nas normas nacionais e internacionais regulamentadoras de pesquisas envolvendo seres humanos. Teve o projeto aprovado pelo Comitê de Ética em Pesquisa da Universidade Estadual de Ponta Grossa (UEPG) no dia 10 de junho de 2016 sob o número: 1.586 .568$.

\section{RESULTADOS}

Fizeram parte da pesquisa 82 (100,0\%) profissionais de saúde e destes foram identificados 39 médicos, 34 enfermeiros e 09 farmacêuticos. A média de idade dos profissionais de saúde foi de 42 anos com idade entre 24 e 65 anos, quanto ao tempo de formação a média foi de 13 anos, entre 08 meses e 40 anos de atuação nas diversas áreas do conhecimento.

Quanto à distribuição por gênero dos profissionais de saúde, apresentada na tabela 1. há predominância do gênero feminino com 57 (69,5\%) profissionais, contabilizando 30 (52,6\%) enfermeiras, 20 (35,1\%) médicas e 7 (12,3\%) farmacêuticas.O gênero masculino corresponde a 25 funcionários (30,5\%), com 19 médicos (76,0\%), 4 enfermeiros (16,0\%) e 2 farmacêuticos (8,0\%).

Do total de 76 (92,7\%) profissionais, afirmaram possuir algum conhecimento sobre a doença celíaca: 37 médicos (48,7\%); 33 enfermeiros (43,4\%) e 6 farmacêuticos (7,9\%). Contudo 12 profissionais $(14,6 \%)$ afirmaram que conheciam o protocolo de

Tabela 1. Distribuição por gênero dos profissionais de saúde na atenção primária à saúde.

\begin{tabular}{lclll}
\hline \multicolumn{2}{c}{ Médico } & Enfermeiro & Farmacêutico & $\mathbf{n}(\%)$ \\
Feminino & $20(51,2 \%)$ & $30(88,2 \%)$ & $7(77,7 \%)$ & $57(69,5 \%)$ \\
Masculino & $19(48,7 \%)$ & $4(11,7 \%)$ & $(22,2 \%)$ & $25(30,4 \%)$ \\
Total & 39 & 34 & 9 & $82(100 \%)$ \\
\hline
\end{tabular}

Fonte: Elaborado pelos autores (2018) 
atendimento aos celíacos do Ministério da Saúde, sendo eles 10 médicos (83,3\%) e 02 enfermeiros $(16,7 \%)$, conforme a tabela 2 . Ressalta-se, nesta pesquisa, que nenhum farmacêutico conhecia o protocolo de atendimento aos celíacos do Ministério da Saúde.

Pode ser observado no Quadro 1, as seis questões que contemplam os requisitos da assistência ao paciente celíaco, e os respectivos acertos dos profissionais sobre a doença. Reconheceram a forma típica da doença 80\% dos pesquisados. Sobre os alimentos que contêm o glúten, 50\% da amostra respondeu corretamente, a classe médica obteve 39\% de acertos.

A sintomatologia da forma não clássica foi identificada por 40\% de acertos. Em relação aos setores em que o glúten é encontrado, 61\% da população estudada respondeu corretamente, a classe farmacêutica representou 6\% de acertos da amostra. Quanto às orientações e cuidados recomendados ao paciente celíaco 88\% assinalaram a opção correta. A definição da contaminação cruzada obteve $57 \%$ de acertos, sendo representada por $44 \%$ dos profissionais da enfermagem.

Tabela 2. Conhecimento dos profissionais de saúde da atenção primária sobre a doença e protocolo de atendimento.

\begin{tabular}{lllll}
\hline & Médico & Enfermeiro & Farmacêutico & Total\% \\
\hline $\begin{array}{l}\text { Possui algum conhecimento sobre a } \\
\text { doença celiaca? }\end{array}$ & & & & \\
Sim & $37(94,8 \%)$ & $33(97 \%)$ & $6(66,6 \%)$ & $92,7 \%$ \\
$\begin{array}{l}\text { Possui conhecimento a respeito do Protocolo } \\
\text { Clínico do atendimento ao paciente celiaco? }\end{array}$ & $10(25,6 \%)$ & $2(5,8 \%)$ & $0(0 \%)$ & $14,6 \%$ \\
$\begin{array}{l}\text { Sim } \\
\text { Total }(\mathbf{n})\end{array}$ & $\mathrm{n}=39$ & $\mathrm{n}=34$ & $\mathrm{n}=9$ & $100 \%$ \\
\hline
\end{tabular}

Fonte: Elaborado pelos autores (2018)

Quadro 1 Distribuição das respostas assertivas sobre a doença celíaca.

\begin{tabular}{l|l}
\hline Questão 1: Tríade de sinais e sintomas da doença em sua forma clássica: & Acertos: 66(80,5\%) \\
$\begin{array}{l}\text { a) Enxaqueca, pirose, diarreia crônica. } \\
\text { b) Dermatite herpetiforme, constipação intestinal, gases. }\end{array}$ & 28 (42,4\%) Enfermes \\
c) Diarreia crônica, perda de peso, distensão abdominal & 06 (9,1\%) Farmacêuticos \\
Questão 2: O glúten é uma proteína que está estritamente relacionada & \\
com a doença celíaca: & Acertos: 41(50,0\%) \\
a) Trigo, aveia, soja, leite, cevada & $16(39,0 \%)$ Médicos \\
b) Aveia, trigo, cevada, malte, centeio & 20 (48,8\%) Enfermeiros \\
c) Cevada, trigo-sarraceno, soja, malte, aveia & 05 (12,2\%) Farmacêuticos
\end{tabular}


Questão 3: A forma não clássica da doença caracteriza-se por:

a) Alterações sorológicas e histológicas do intestino delgado, na ausência de manifestações clínicas.

b) Por um quadro de sintomas em que manifestações digestivas estão ausentes, ou quando presentes, ocupam um segundo plano.

c) Crise celíaca, sua forma clínica pode ter uma evolução grave sendo desencadeada por uma infecção, que ocorre quando há retardo no diagnóstico e consequentemente no tratamento.

Questão 4: Em quais setores o glúten pode estar inserido:
a) Alimentício apenas.
b) Alimentício e cosmético.
c) Cosmético, alimentício e farmacêutico.

Questão 5: Que orientações e cuidados são recomendados ao paciente celíaco:

a) Dieta restritiva, eliminando totalmente o glúten da alimentação.

b) Reduzir o consumo de glúten, sem a necessidade da exclusão total desta proteína em sua dieta.

c) Tratar com medicamentos específicos, uma alimentação saudável com boa ingesta hídrica.

Questão 6: Em Quando acontece a contaminação cruzada:

a) Trata-se da condição de risco do indivíduo relacionada à prevalência da doença na família, um parente de $1^{\circ}$ grau.

b) Alimentos manipulados e armazenados em locais que contenham vestígios do glúten.

c) Transmissão da doença por contato de fluidos sanguíneos de um celíaco, predispondo outro a desenvolver a doença.
Acertos: 33(40,2\%)

19 (57,6\%) Médicos

$11(33,3 \%)$ Enfermeiros

03 (9,1\%) Farmacêuticos

\section{Acertos: 50 (60,9\%)}

24 (48,0\%) Médicos

23 (46,0\%) Enfermeiros

03 (6,0\%) Farmacêuticos

Acertos: 72 (87,8\%)

32 (44,4\%) Médicos

32 (44,4\%) Enfermeiros

08 (11,1\%) Farmacêuticos

Acertos: $47(57,3 \%)$

27 (57,5\%) Médicos

15 (31,9\%) Enfermeiros

05(10,6\%) Farmacêuticos

Fonte: Elaborado pelos autores (2018)

\section{DISCUSSÃO}

Verificou-se o predomínio do sexo feminino na pesquisa, principalmente na enfermagem. Diante da amplitude e complexidade que envolvem estudar o cenário multiprofissional da saúde, a enfermagem é analisada como prática naturalizada feminina, provavelmente uma das profissões mais antigas exercidas por mulheres ${ }^{9}$.

Em relação ao conhecimento dos profissionais de saúde sobre fisiopatologia da Doença Celíaca, representou 92,7\% das respostas assertivas. Já o entendimento do Protocolo sobre as Diretrizes Terapêuticas foi de $14,6 \%$. Os dados da pesquisa evidenciaram que a maioria dos profissionais desconhece sobre o protocolo de atendimento. Compreende-se, após análise destes dados, que o conhecimento dos profissionais especificamente sobre a doença considera-se maior em referência às diretrizes terapêuticas. Ressalta-se que o desconhecimento pode impactar na identificação precoce e prevenção de complicações que a doença pode manifestar. 
Informações nacionais apontam que há fragilidade no diagnóstico sendo considerada uma doença negligenciada, e devido a este fato mencionam que a Doença Celíaca possui baixa prevalência, incidência e mortalidade ${ }^{10}$.

Pesquisa ${ }^{11}$ reforça que nos últimos anos o conhecimento epidemiológico da Doença Celíaca cresceu significativamente devido à identificação da grande variedade de manifestações clínicas dessa doença. Isto sugere a importância da capacitação dos profissionais de saúde, tratando-se de um processo educacional de curto prazo, envolvendo todas as ações que visam deliberadamente ampliar a capacidade das pessoas para desempenhar melhor as atividades relacionadas ao cargo de suas responsabilidades ${ }^{12}$.

Pode-se afirmar que a educação em serviço possibilita o aprimoramento da formação profissional por meio de capacitações. Ações de promoção à saúde devem ser estimuladas mediante a utilização do protocolo clínico com intenção de fornecer a assistência prestada de qualidade ${ }^{13}$.

Sobre os questionamentos relacionados à patogenia, a doença foi definida como desordem do sistema imunológico pela maioria dos profissionais de saúde. Estudos conceituam a Doença Celíaca como sendo autoimune, acometendo indivíduos geneticamente predispostos à intolerância ao glúten. O diagnóstico baseia-se em testes sorológicos para anticorpos contra transglutaminase tecidual (TTG) e peptídeos de gliadina desamidados ${ }^{14}$.

A resposta prevalente entre os profissionais sobre qual órgão a doença celíaca acomete foi o intestino. 0 órgão afetado apresenta vilosidades achatadas na mucosa devido à inflamação, pela qual é induzida em pessoas geneticamente suscetiveis pela ingestão de proteínas do trigo, centeio e cevada que contêm glúten ${ }^{15}$.

Quanto aos fatores que predispõem o indivíduo a desenvolver a patologia, uma porção mínima da população estudada reconheceu o fator ambiental como componente. No entanto, a maior parte da amostra demonstrou ciência do fator genético e imunológico, outros fatores predispostos. A condição celíaca representa uma forte condição hereditária, constituindo-se numa enfermidade multifatorial, envolvendo tanto componentes genéticos, como ambientais. A combinação de alguns fatores induz à patologia intestinal. Uma pesquisa realizada nos Estados Unidos constatou a predominância de familiares de $1^{\circ}$ grau com a Doença Celíaca e a possibilidade do teste genético para identificar tal doença, por sua vez, esse teste ainda é restrito pelo alto custo ${ }^{16-17}$.

Os três fatores que predispõem o indivíduo à patologia são: ambiental, o glúten como fator indutor da doença; genético, cerca de 90,0-95,0\% dos doentes expressam moléculas de um complexo responsável por importante papel no sistema imune; e imunológico, devido à resposta imunitária anormal aos derivados da gliadina e da glutenina ${ }^{18}$.

A forma típica/clássica da doença foi reconhecida por $80,5 \%$ dos profissionais, isso se deve ao fato que a maioria dos indivíduos possui a forma clássica da doença, onde a identificação dos sintomas são mais comuns. Atualmente manifestações extra-intestinais, tais como anemia por deficiência de ferro, crescimento vacilante, puberdade tardia e úlceras na boca, estão sendo cada vez mais reconhecidas em crianças e adolescentes do Reino Unido ${ }^{6,19}$.

Ao contrário da forma típica, a manifestação atípica/não clássica obteve apenas 40,2\% de acertos. É de extrema importância que os profissionais de saúde estejam atentos para esta manifestação como possivel forma de apresentação da doença, e, em caso de suspeita, deve-se realizar uma investigação pertinente ${ }^{20}$.

Referente à presença do glúten nos alimentos, $50,0 \%$ dos profissionais assinalaram corretamente, sendo que ele corresponde à principal fração proteica presente no trigo, no centeio, na cevada e na aveia. Desta população apenas 39,0\% da classe médica esteve ciente dos alimentos que possuem glúten. Os 5,0\% da amostra que não responderam à questão foram médicos, sendo esta classe predominante na pesquisa. Há importância do conhecimento do paciente em relação à doença 
e seu tratamento, e para isto os médicos têm a responsabilidade de esclarecê-los da forma mais detalhada possivel. Os conhecimentos transmitidos por estes profissionais é um fator importante para garantir o sucesso do tratamento do paciente ${ }^{6}$.

A respeito dos setores em que o glúten está presente, 60,9\% assinalaram a opção correta como os setores alimentício, cosmético e farmacêutico. 0 glúten está presente como um elemento cultural adicionado não só em alimentos, mas também nos seus subprodutos. Apenas 6,0\% dos farmacêuticos estavam cientes da presença do glúten no setor farmacêutico, e vale ressaltar que, devido à terapêutica da condição celíaca ser essencialmente dietética, percebe-se um desinteresse comercial por parte da indústria farmacêutica em apoiar e financiar a divulgação ou formação de profissionais atuantes $^{14}$.

Os profissionais enfermeiros têm como competências educar o paciente sobre a complexidade da dieta, as diversas fontes de glúten e alimentos passíveis de contaminação cruzada, informar a disponibilidade de produtos sem glúten no mercado e a correta orientação nutricional21. Dessa forma, reitera-se a importância do manejo dietético adequado que contribua a melhora dos sintomas apresentada por esses indivíduos 22 .

Com relação à contaminação cruzada, $57,3 \%$ dos profissionais de saúde responderam corretamente. Diante destes resultados foi constatado pouco conhecimento das equipes de saúde a respeito da Doença Celíaca e, principalmente, dos riscos associados à contaminação cruzada por glúten. Compreender a contaminação cruzada, a eliminação dos resíduos e traços de glúten faz parte da iniciação na vida de um celíaco ${ }^{10}$.

Uma das formas de ter o conhecimento é por meio de capacitações, buscando melhoria da assistência. Desse modo, a pesquisadora participante ministrou uma palestra sobre a Doença Celíaca para usuários do Sistema Único de Saúde e profissionais de uma Unidade Básica de Saúde. Foram elaborados e distribuídos folders com as principais informações da Doença Celíaca e receitas alimentares sem glúten. A temática despertou interesse dos profissionais, e dúvidas foram esclarecidas.

\section{CONCLUSÃO}

Conclui-se que o conhecimento dos profissionais de saúde relacionado à Doença Celíaca, quanto à sintomatologia, necessita ser aprofundado frente ao protocolo das diretrizes de assistência ao paciente. Pois o conhecimento superficial, pelos profissionais de saúde, implica na demora da captação dos pacientes não diagnosticados bem como nas orientações ao paciente celíaco quanto ao tratamento a ser seguido.

Sugere-se, por meio deste estudo, implementar a educação em serviço como estratégia de elencar discussões que são importantes para o direcionamento da conduta clínica.

\section{REFERÊNCIAS}

1. Ministério da Saúde (BR). Secretaria de Atenção à Saúde. Portaria $n^{\circ} 1149$, de 11 de novembro de 2015: aprova o protocolo clínico e diretrizes terapêuticas da doença celíaca. Brasilia: Ministério da Saúde: 2015.

2. Nadal J, Ferreira SMR, Costa IB da, Schmidt ST. O princípio do direito humano à alimentação adequada e a doença celíaca: avanços e desafios. Demetra [Internet]. 2013 [citado 2018 Out 22]: 8(3):411-23. Disponivel em: http://www.fenacelbra.com.br/ arquivos/publicacoes/principio_direito_humano_alimentacao.pdf

3. Magalhães JP, Oliveira MS, Monteiro MAM, Schaefer, MA, Garcia MAVT. Riscos de contaminação por glúten em um restaurante universitário com preparações para indivíduos celíacos: um estudo de caso. Nutr Clín Diet Hosp. 2017; 37(1):165-70.

4.Rocha S, Gandolfi L, Santos JE dos. The psychosocial impacts caused by diagnosis and treatment of Coeliac Disease. Rev Esc Enferm USP. 2016; 50(1):66-72. doi: http://dx.doi.org/10.1590/ S0080-6234201c60000100009

5. Araújo HMC, Araújo WMC, Botelho RBA, Zandonadi RP. Doença celíaca, hábitos e práticas alimentares e qualidade de vida. Rev Nutr. 2010; 23(3): 467-74. doi: http://dx.doi.org/10.1590/S141552732010000300014

6. Sdepanian VL, Morais MB de, Neto UF. Doença celíaca: avaliação da obediência a dieta isenta de glúten e do conhecimento da doença pelos pacientes cadastrados na Associação dos Celíacos 
do Brasil (ACELBRA). Arq. Gastroenterol. 2001;38(4):232-39. doi: http://dx.doi.org/10.1590/S0004-28032001000400005.

7. Medina MG, Aquino R, Vilasbôas ALQ, Mota E, Pinto Junior EP, Luz LA da, et al. Promoção da saúde e prevenção de doenças crônicas: o que fazem as equipes de saúde da família?. Saúde em debate. 2014:38(spe):69-82. doi: http://dx.doi.org/10.5935/01031104.20145006

8. Ministério da Saúde (BR). Secretaria Municipal de Ponta Grossa. Plano Municipal de Saúde Ponta Grossa 2018 /2021. Ponta Grossa: Ministério da Saúde: 2017.

9. Ojeda BS, Eidt OR, Canabarro S, Corbellini VL, Creutzberg M. Saberes e verdades acerca da enfermagem: discursos de alunos ingressantes. Rev Bras. Enferm. [Internet] 2008 [citado em 2016 out 26];61(1):78-84. doi: http://dx.doi.org/10.1590/S003471672008000100012

10. Paula FA de, Crucinsky J. Benati R. Fragilidades da atenção à saúde de pessoas celíacas no Sistema Único de Saúde (SUS): a perspectiva do usuário. Demetra. 2014:9 (supl 1):311-28. doi: http:// dx.doi.org/10.12957/demetra.2014.10499

11. Oliveira A, Trindade E, Tavares M, Lima R, Terra M, Dias JA. Celiac disease in first degree relatives of celiac children. Arq. Gastroenterol.2012;49(3):204-207. doi: http://dx.doi.org/10.1590/ S0004-28032012000300007

12. Leandro AIP, Branco ES. Importância do treinamento e desenvolvimento nos serviços de saúde. Rev Adm Hosp Inov Saúde 2011;(6):64-69. doi: http://dx.doi.org/10.21450/rahis. v0i6.1018.

13. Ministério da Saúde (BR). Secretaria de Gestão do Trabalho e da Educação na Saúde. Política Nacional de Educação Permanente. Brasília: Ministério da Saúde; 2009.

14. Ludvigsson JF, Leffler DA, Bai JC, Biagi F, Fasano A, Green PHR, et al. The Oslo definitions for coeliac disease and related terms. Gut [Internet]. 2013 [cited 2016 fev. 20]; 2(1):43-52. doi: http://gut.bmj.com/content/gutjnl/62/1/43.full.pdf

15. Pelleboer RAA, Janssen RLH, Deckers-Kocken JM, Wouters E, Nissen AC, Bolz WEA, et al. Celiac disease is overrepresented in patients with constipation. J Pediatr (Rio J).2012:88(2):173-6. doi: http://dx.doi.org/10.2223/JPED.2155

16. Rosa Utiyama SR da, Messias Reason IJT de, Silva Kotze LM da. Aspectos genéticos e imunopatogênicos da doença celíaca: visão atual. Arq. Gastroenterol. [Internet];2004:41(2):121-28. doi: http://dx.doi.org/10.1590/S0004-28032004000200010.

17. Roy A, Pallai M, Lebwohl B, Taylor AK, Green PH. Attitudes toward genetic testing for celiac disease. J Genet Counsel.2016;25(2):270-8.doi:http://dx.doi.org/10.1007/s10897015-9867-z.

18. Nobre SR, Silva T, Cabral JEP. Doença celíaca revisitada. Jornal Português de Gastrenterologia, Lisboa 2007:14(4):184-93.

19. Paul SP. McVeigh L, Gil-Zaragozano E, Basude D. Diagnosis and nursing management of coeliac disease in children. Nurs Child Young People 2016 Feb;28(1):18-24. doi: 10.7748/ncyp.28.1.18.s28.

20. Boé, C, Lozinsky AC, Patrício FR, Andrade JAB de, Fagundes Neto U. Doença celíaca e constipação: uma manifestação clínica atípica e pouco frequente. Rev Paul Pediatr. 2012 Jun;30(2):28387.

21. Abreu LCS de. A importância da enfermagem no tratamento de doenças celíacas. Web Artigos [Internet]. 2015 [citado 2018 Out 10]. Disponivel em: http://www.webartigos.com/ resources/ files/_modules/article/article_136789_201510191700426dda.pdf
22. Melo Ribeiro PV de, Paula Santos A de, Andreoli CS, Ribeiro SMR, Paula Jorge M de, Moreira AVB. Nutritional status variation and intestinal and extra intestinal symptomatology in patients with celiac disease and non-celiac gluten sensitivity given specialized dietary advice. Rev Nutr. 2017:30(1):57-67.doi: http:// dx.doi.org/10.1590/1678-98652017000100006 